\title{
Optical properties of AlN determined by vacuum ultraviolet spectroscopy and spectroscopic ellipsometry data
}

\author{
D.J. Jones and R.H. French ${ }^{\text {a) }}$ \\ DuPont Central Research, E356-384 Experimental Station, Wilmington, Delaware 19880 \\ H. Müllejans ${ }^{\text {b) }}$ \\ Max-Planck-Institut für Metallforschung, Seestraße 92, D-70174 Stuttgart, Germany \\ S. Loughin \\ DuPont Central Research, E356-384 Experimental Station, Wilmington, Delaware 19880 \\ A.D. Dorneich \\ Max-Planck-Institut für Metallforschung, Seestraße 92, D-70174 Stuttgart, Germany \\ P.F. Carcia \\ DuPont Central Research, E356-384 Experimental Station, Wilmington, Delaware 19880
}

(Received 2 November 1998; accepted 3 August 1999)

\begin{abstract}
Precise and accurate knowledge of the optical properties of aluminum nitride (AlN) in the ultraviolet (UV) and visible (VIS) regions is important because of the increasing application of AIN in optical and electro-optical devices, including compact disks, phase shift lithography masks, and AIN/GaN multilayer devices. The interband optical properties in the vacuum ultraviolet (VUV) region of $6-44 \mathrm{eV}$ have been investigated previously because they convey detailed information on the electronic structure and interatomic bonding of the material. In this work, we have combined spectroscopic ellipsometry with UV/VIS and VUV spectroscopy to directly determine the optical constants of AlN in this range, thereby reducing the uncertainty in the preparation of the low-energy data extrapolation essential for Kramers-Kronig analysis of VUV reflectance. We report the complex optical properties of AlN, over the range of $1.5-42 \mathrm{eV}$, showing improved agreement with theory when contrasted with earlier results.
\end{abstract}

\section{INTRODUCTION}

Interest in aluminum nitride (AIN) has increased ever since Slack ${ }^{1}$ and McNelly ${ }^{2,3}$ synthesized high-purity, high thermal conductivity, single crystals. This material has found many technological applications ${ }^{4}$ motivating considerable study in theoretical ${ }^{5-13}$ and experimental ${ }^{14}$ investigations. Due to its high intrinsic thermal conductivity, AlN is attractive as an electronic packaging substrate. AlN is also used as a phase contrast layer in optical disks and has potential as a phase shift material in lithographic photo masks. ${ }^{15,16} \mathrm{AlN} / \mathrm{GaN}$ alloys and multilayers find roles in optical devices such as blue lasers. These applications generate an interest in the optical properties and electronic structure of AlN. The literature on AlN spans vibrational spectroscopy, ${ }^{17}$ ultraviolet

\footnotetext{
a) Address all correspondence to this author.

e-mail: roger.h.french@usa.dupont.com

b) Present address: Institute for Advanced Materials, Joint Research Centre, European Commission, PO Box 2, NL-1755 ZG Petten, The Netherlands.
}

(UV) spectroscopy in the band gap region ${ }^{18-27}$ including temperature-dependent studies ${ }^{28}$ and polarization dependent studies, ${ }^{20,29}$ a number of vacuum ultraviolet (VUV) studies, ${ }^{30,37}$ electron energy loss spectroscopy (EELS) studies, ${ }^{32,33}$ although in most cases these results are consistent with oxidized material, ${ }^{34}$ and general reviews. ${ }^{35,36}$

Knowledge of the optical properties is significant not only for optical applications but also for providing insight into the electronic band structure of a material. In previous work, this group employed VUV spectroscopy $^{37}$ to compare the measured electronic structure of AlN with theoretical band structure calculations. Here, we augment the VUV reflectance measurements over the photon energy range of $2-40 \mathrm{eV}$ with spectroscopic ellipsometry measurements over the range of $1.5-6 \mathrm{eV}$. A procedure for simultaneously performing KramersKronig dispersion analysis on the data from these two sources is described in the Appendix. The augmented data span a wider energy range with an improved accuracy in amplitude, affording greater precision in determining the quantitative optical properties of AlN. The result is that the optical properties of AlN as determined in this work are in better agreement with theory. 


\section{EXPERIMENTAL METHODS}

\section{A. Sample preparation}

Thin film samples were used for the ellipsometric and UV/VIS studies, while the VUV spectroscopy was performed on a bulk single crystal, grown by Slack, ${ }^{1}$ who reported thermal conductivity of $275 \mathrm{~W} \mathrm{~m}^{-1} \mathrm{~K}^{-1}$ and oxygen concentration of $340 \mathrm{ppm}$. The VUV specimen was oriented with the $c$ axis normal $(\mathbf{E} \perp \mathbf{c})$ and polished nonaqueously to achieve a nearly oxygen free surface, as described elsewhere. ${ }^{37}$ The AlN thin films were formed by reactive sputtering from an $\mathrm{Al}$ target in a partial pressure of $\mathrm{N}_{2}$ in Ar. Sputtering at intermediate partial pressure produced a mixture of $\mathrm{AlN}$ and $\mathrm{Al}$ phases. The target was 3-in. diameter $\mathrm{Al}$ and was sputtered with a low-profile, radio-frequency (rf) magnetron sputtering gun in a MRC 8667 sputtering system. Prior to sputtering films, the Al target was presputtered in 10 mtorr Ar at $500 \mathrm{~W}$ for $1 \mathrm{~h}$ to obtain a clean and highly reactive target surface. The deposition conditions for the AlN thin film \#8346 consisted of a total pressure of $\mathrm{Ar}+\mathrm{N}_{2}$ of 5.22 mtorr, an $\mathrm{N}_{2} / \mathrm{Ar}+\mathrm{N}_{2}$ fraction of $20 \%$, with $500 \mathrm{~W}$ of rf at $320 \mathrm{~V}$ applied to the target. The resultant deposition rate was $0.3 \mathrm{~nm} / \mathrm{s}$. AlN film \#8346 was single phase AlN with a nominal thickness of $100 \mathrm{~nm}$, exhibiting wurtzite structure and $c$-axis texture on the basis of $\mathrm{x}$-ray diffraction. The films were deposited on ultra-highpurity fused quartz (glass) typically used as lithographic photomask substrates. ${ }^{38}$

\section{B. Spectroscopy}

Spectroscopic ellipsometry was performed with the Woollam 193/DUV instrument, which has a range from 1.13 to $6.67 \mathrm{eV}$ (1100 to $186 \mathrm{~nm}$ ), and employs $\mathrm{MgF}_{2}$ polarizers and analyzers rather than the more common calcite optics. The spot diameter at the sample is $2 \mathrm{~mm}$. The instrument measures the ellipsometric parameters $\Psi$ and $\Delta$, defined by the equation

$$
\tan (\Psi) e^{\Delta \cdot i}=\frac{R_{P}}{R_{S}}
$$

where $R_{P} / R_{S}$ is the complex ratio of the $p$ - and $s$ polarized components of the reflected amplitudes. These parameters are analyzed using the Fresnel equations ${ }^{39}$ in a computer-based modeling technique to directly determine the thin film's optical constants.

Ellipsometric measurements were conducted using light incident on the front surface of the film. This sample configuration requires the back surface of the quartz substrate to be mechanically roughened to eliminate the depolarizing effects of back-surface reflections in the substrate. Front side ellipsometric measurements
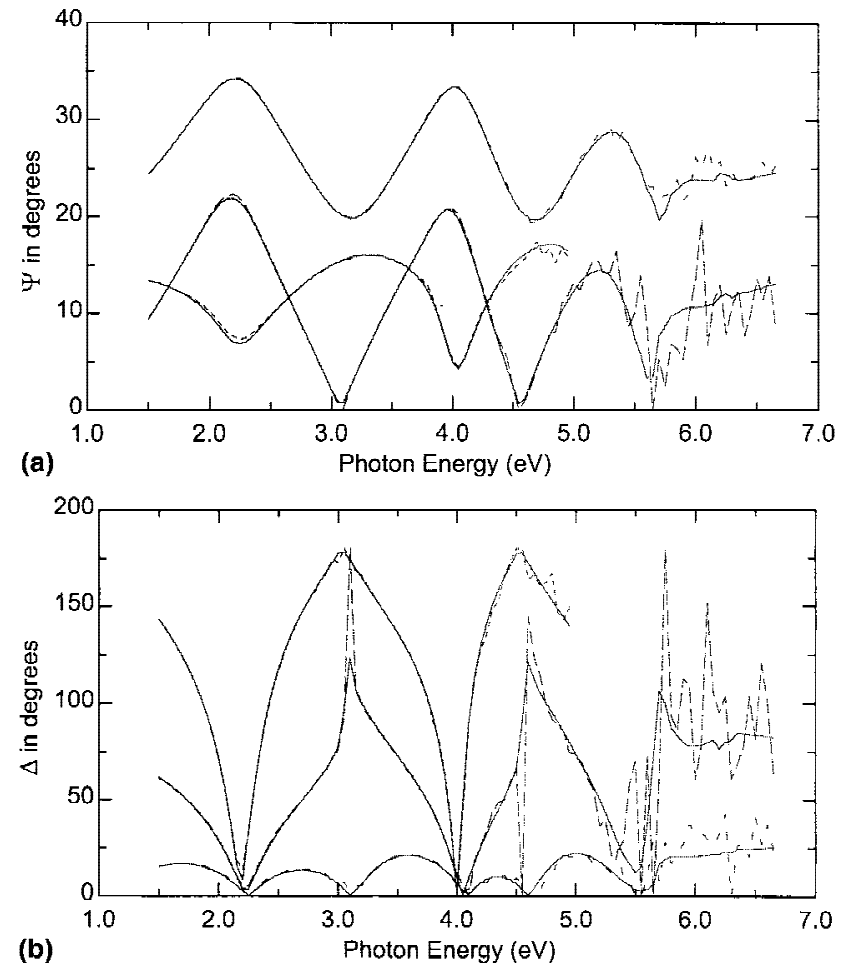

FIG. 1. Ellipsometric data for parameters (a) $\Psi$ and (b) $\Delta$ data for thin-film AlN deposited in $\mathrm{Ar}+20 \% \mathrm{~N}_{2}$ sputtering gas. The dashed line is the data and the solid line is the model fit for angles of incidence of $60^{\circ}, 70^{\circ}$, and $80^{\circ}$.

on samples in this configuration were obtained with light incident at an angle of $60^{\circ}, 70^{\circ}$, and $80^{\circ}$ relative to the film normal. The ellipsometric results for the AlN \#8346 are shown in Fig. 1.

The 193/DUV can also determine the normal incidence film transmission, integrated over an area of 2-mm diameter. The transmission of the thin film AlN sample measured on the ellipsometer is compared with the UV/ visible (VIS) spectrophotometer measurement shown in Fig. 2(a), to verify reproducibility and avoid any experimental artifacts. Using transmission data in the optical analysis reduces the effective surface sensitivity of ellipsometry alone, ensuring that bulk properties of the film, such as the extinction or absorption, will be fully accounted for in the Fresnel analysis.

A Perkin-Elmer Lambda 9 spectrophotometer, with transmission and reflectance attachments, is used to cover the UV/VIS near infrared (NIR) spectral regions. This tool employs a V-W absolute reflectance attachment with both xenon and deuterium lamps. The transmission of smooth-back samples, as shown in Fig. 2(a), and the reflection of rough-back samples, as shown in Fig. 2(b), are both measured on this spectrophotometer. Reflectance of samples in the $\mathrm{W}$ configuration was measured with the first reflection from the sample and the second 
reflection from an aluminum reference mirror. The results were corrected for this reference mirror reflectance to determine the reflectance of the sample.

The VUV instrument used in this work has a range of 2-40 eV (620-31 nm) and has been previously described. ${ }^{40,41}$ The VUV reflectance data employed in this work have been reported elsewhere. However, by augmenting these data with the ellipsometric measurements, we have obtained more accurate results for the quantitative optical properties that give substantially better agreement with theory as described in the next section.

\section{RESULTS}

\section{A. Analysis of ellipsometry data}

To determine the optical functions of the thin film samples, we use both ellipsometric and UV/VIS transmission and reflection data taken on the same film to find a solution of the model describing the optical behavior of the thin-film/substrate system. ${ }^{39}$ The variable angle of incidence spectroscopic ellipsometry (VASE) software analyzes experimental ellipsometric and UV/VIS data, with respect to an adjustable parameter model, to generate a viable optical model comprising microstructural parameters and material optical constants for the thinfilm system.
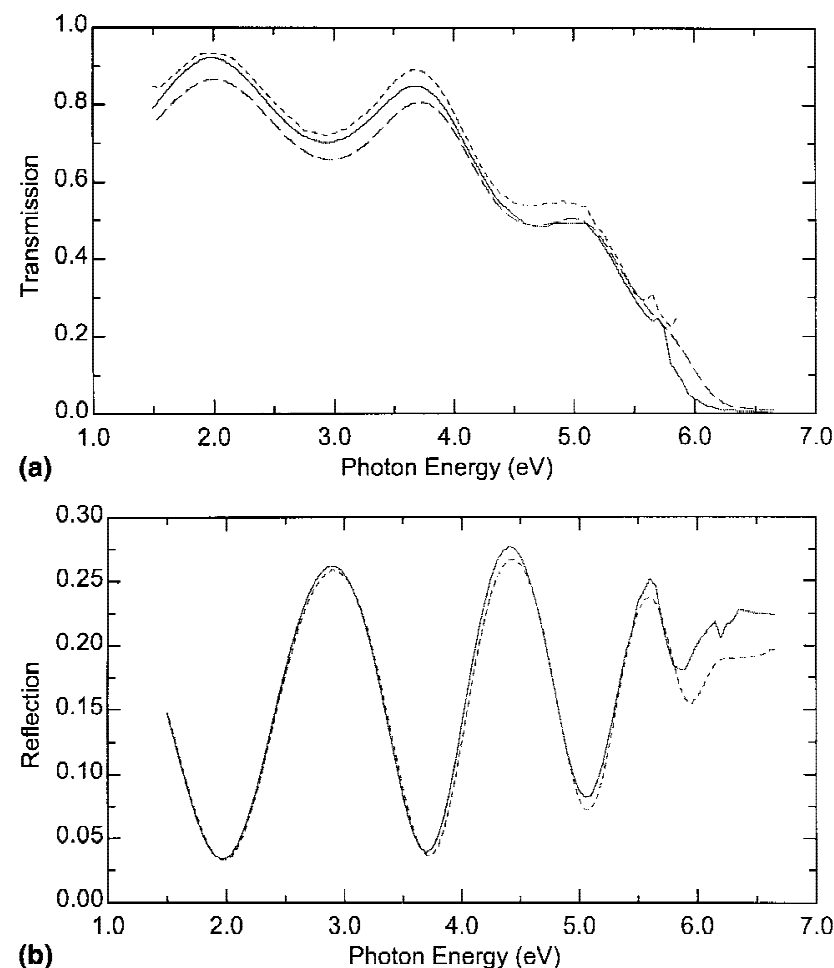

FIG. 2. UV/VIS transmission data for (a) smooth-back samples and reflectance data for (b) rough-back samples of thin-film AlN deposited in $\mathrm{Ar}+20 \% \mathrm{~N}_{2}$ sputtering gas. The dashed line is the data and the solid line is the model fit.
The thin-film AlN sample \#8346 was modeled as a homogeneous thin film with surface and substrate interfaces represented by an effective medium approximation (EMA) layer of 50\% AlN and 50\% voids. By nonlinear regression, the model was fit to the experimental data. The refined model thicknesses were $55.56 \AA$ for the surface EMA, $1484.5 \AA$ for the film, and $0 \AA$ for the film/ substrate EMA, with a substrate thickness of $2.286 \mathrm{~mm}$. The complex index of refraction for this solution is shown in Fig. 3.

\section{B. Optical properties}

As described in the Appendix, after $R(E)$ is appropriately scaled and augmented, a Kramers-Kronig analysis recovers the phase, from which the index of refraction for single-crystal AIN is calculated and shown in Fig. 4. The

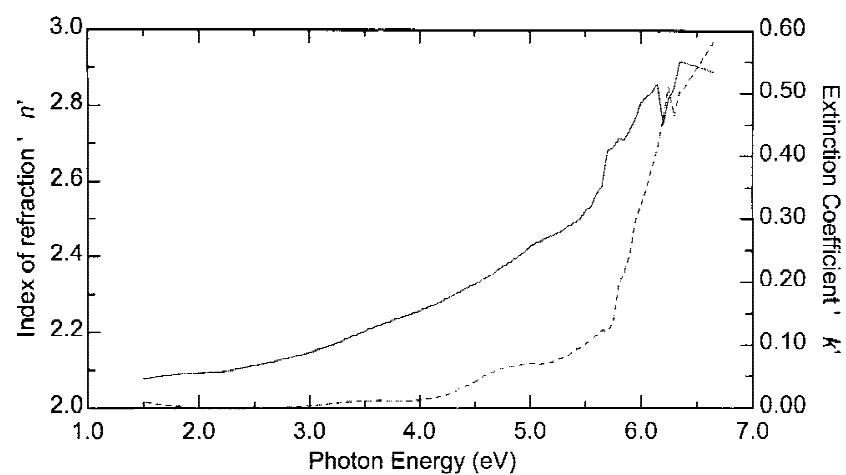

FIG. 3. Complex index of refraction, $\hat{n}=n+i k$, determined for thin-film AlN deposited in $\mathrm{Ar}+20 \% \mathrm{~N}_{2}$ sputtering gas. $n$ is the solid line, while $k$ is the dashed line.

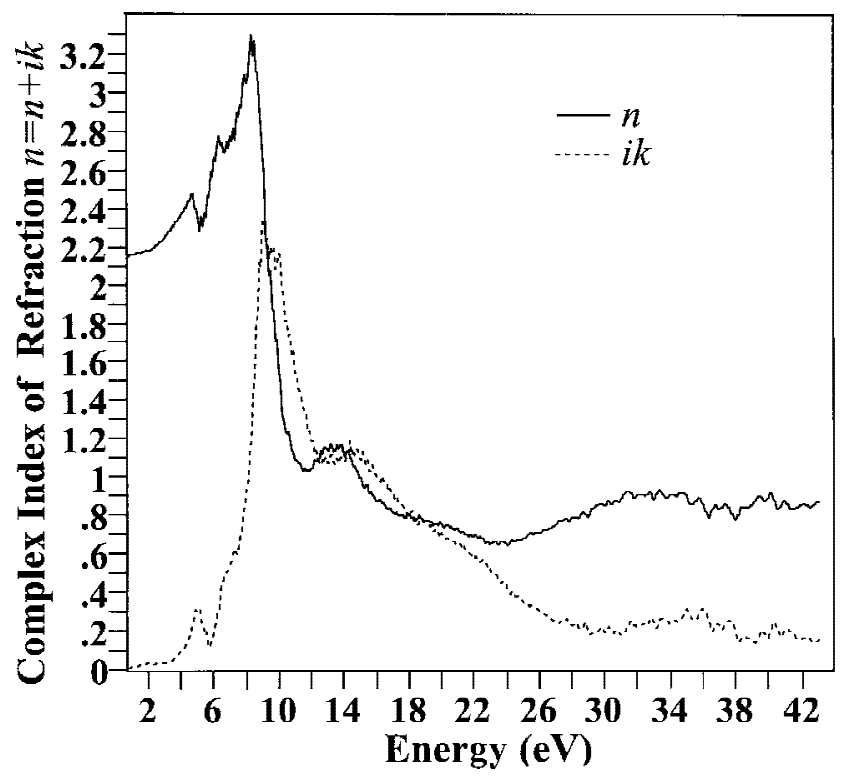

FIG. 4. Complex index of refraction, $\hat{n}=n+i k$ of single-crystal AlN determined for light polarized $\mathbf{E} \perp \mathbf{c}$, where $n$ is shown by the solid line and $k$ by the dashed line. 

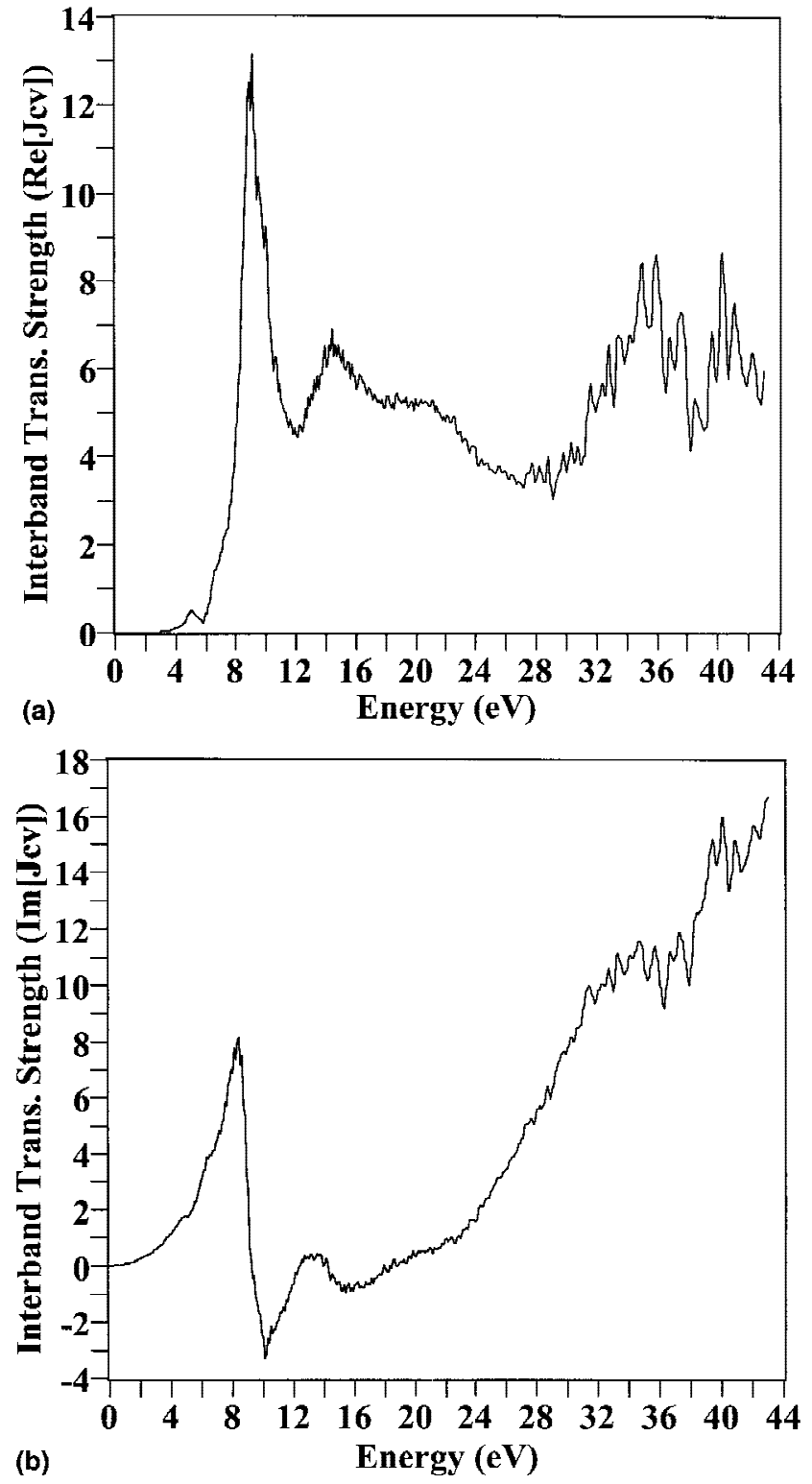

FIG. 5. (a) Real and (b) imaginary parts of the interband transitions strength of single-crystal AlN determined from Kramers-Kronig analysis of VUV reflectance data.

dielectric function is obtained from the relation $\epsilon_{1}+i \epsilon_{2}=$ $(n+i k)^{2}$. Here we render the optical response in terms of the interband transition strength, $J_{\mathrm{cv}}(E)$, related to $\epsilon(\omega)$ by

$$
J_{\mathrm{cv}}(E)=\frac{m_{\mathrm{o}}{ }^{2}}{\mathrm{e}^{2} \hbar^{2}} \frac{E^{2}}{8 \pi^{2}}\left(\epsilon_{2}(E)+i \epsilon_{1}(E)\right)
$$

where $J_{\mathrm{cv}}(E)$ is proportional to the transition probability and has units of $\mathrm{g} \mathrm{cm}^{-3}$. For computational convenience we take the prefactor $m_{\mathrm{o}}{ }^{2} \mathrm{e}^{-2} \hbar^{-2}$ in Eq. (2), whose value in cgs units is $8.289 \times 10^{-6} \mathrm{~g} \mathrm{~cm}^{-3} \mathrm{eV}^{-2}$, as unity. Therefore the units of the $J_{\mathrm{cv}}(E)$ spectra shown in Fig. 5 are $\mathrm{eV}^{2}$.

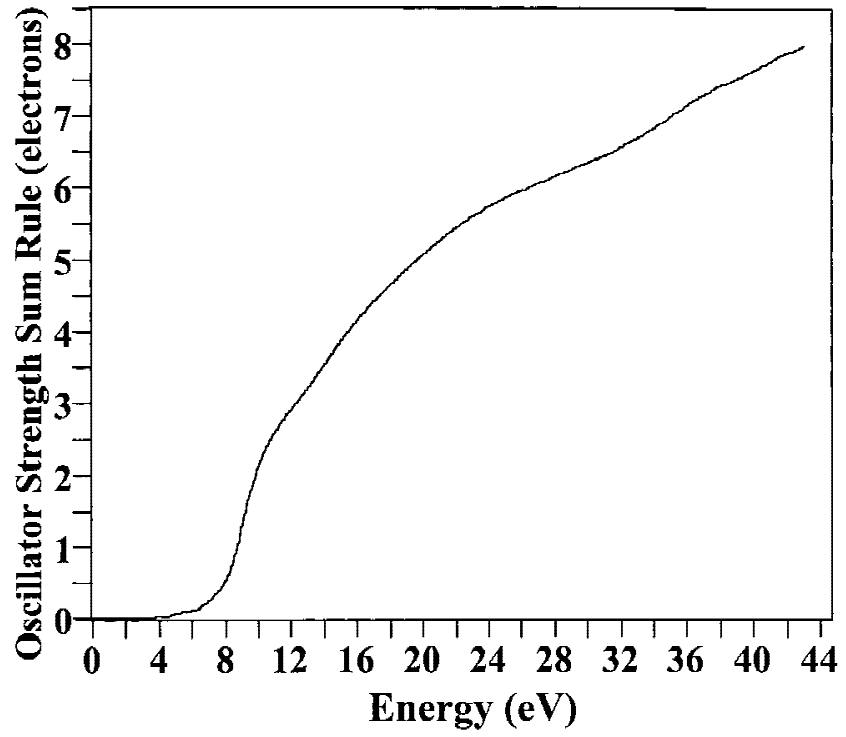

FIG. 6. Oscillator strength sum rule of single-crystal AlN.

With application of the oscillator strength sum rule $e^{42}$ for the interband transition strength, $n_{\text {eff }}(E)$, the number of electrons contributing to a transition up to an energy $E$ is

$$
n_{\text {eff }}(E)=\frac{4 v_{f}}{m_{\mathrm{o}}} \int_{0}^{E} \frac{J_{\mathrm{cv}}\left(E^{\prime}\right)}{E^{\prime}} \mathrm{d} E^{\prime}
$$

where $v_{f}$ is the volume of the AlN formula unit, $20.86 \AA^{3}$. The effective number of electrons participating in interband transitions at energies less than or equal to the photon energy, $E$, based on the oscillator strength sum rule, is displayed for single-crystal AlN in Fig. 6.

\section{DISCUSSION}

\section{A. Optical properties of aluminum nitride}

The optical properties of AlN obtained in this analysis agree qualitatively with the bulk of the prior literature, especially if one compares only the energy location of features. However, the amplitudes of the various features, which are of critical importance to quantitative descriptions, are substantially different. Comparing with our own prior results, ${ }^{37}$ we find that the most significant differences are the relative decrease in amplitude of the low-energy features and the concomitant increase in amplitude of high-energy features. This places the experimental results in much closer agreement with optical properties calculated from first principles. ${ }^{37}$ In the prior work, the N2 $p$ to Al3s transitions, characterized as a balanced set of transitions in the range of 6.2-10.4 eV, dominated the experimental interband transition strength, while the calculated results suggested they should be comparable to the second set in the range of $10.2-25.7 \mathrm{eV}$, arising from transitions from 
TABLE I. Optical constants of aluminum nitride at lithographic wavelengths.

\begin{tabular}{lcc}
\hline \hline Wavelength $(\mathrm{nm})$ & Index of refraction, $n$ & Extinction coefficient, $k$ \\
\hline 248 (DUV) & 2.43 & 0.07 \\
193 & 2.91 & 0.52 \\
\hline
\end{tabular}

hybridized $\mathrm{Al}=\mathrm{N}$ orbitals to $\mathrm{Al} 3 p$ orbitals. In the present work, the first set of transitions are still the most prominent but are much closer in amplitude to the second set. Also, the oscillator-strength sum rule, shown in Fig. 6, indicates that 8 electrons per formula unit participate in transitions at energies at or below $44 \mathrm{eV}$. In previous work, ${ }^{43}$ the value obtained was about 7 electrons participating in transitions at energies at or below $40 \mathrm{eV}$.

One additional point of interest is the low-energy feature at $5.5 \mathrm{eV}$, which is below the band gap energy of $6.2 \mathrm{eV}$. This peak was found in the VUV reflectance and in the spectrophotometric data as well and is attributed to localized electronic states due to vacancies. Accordingly, the low-energy cut-off was selected so as to include this feature.

\section{B. Technological implications}

Because AlN is a candidate material for the attenuating phase-shift layers in embedded phase-shifting photomasks, ${ }^{15,16}$ the complex index of refraction of AlN is of topical interest. This is presented in Table I for two lithographic wavelengths. Thus, for the design of an AlN attenuating, embedded phase shifter for applications at $\lambda=193 \mathrm{~nm}$, a film thickness of $51.9 \mathrm{~nm}$ would produce a $180^{\circ}$ phase shift with $10.6 \%$ transmission. Such precise determination of thickness requirements is enabled by the quantitative results presented here, because precise knowledge of both feature energy and amplitude is required for accurate design.

\section{CONCLUSIONS}

As we have seen, the Kramers-Kronig (KK) dispersion analysis of reflectance data is the critical step in accurately determining the VUV optical properties, but the KK analysis admits some subjective interpretation of the data due to the requirement that measured data be extrapolated to span an infinite energy range. By combining spectroscopic ellipsometry and UV/VIS spectroscopy to directly determining the UV and visible optical properties, we have dramatically reduced the uncertainty in the low-energy extrapolation employed in the KK analysis. This quantitative Kramers-Kronig dispersion analysis of VUV/UV/VIS optical reflectance data provides definitive values for the complex optical properties of AlN over the range of 1.5 to $42 \mathrm{eV}$, which are especially important for emerging AlN applications such as phase shift lithography.

\section{ACKNOWLEDGMENTS}

The authors express their appreciation to Glen A. Slack for the use of the single-crystal AlN sample and to Manfred Rühle for several helpful discussions. The assistance of M.F. Lemon and M. Reilly with the spectroscopic ellipsometry is also appreciated. We are grateful to Dr. L.K. DeNoyer for software development. H.M. acknowledges the financial support of VolkswagenStiftung (Contract I/70 082).

\section{REFERENCES}

1. G.A. Slack, J. Phys. Chem. Solids 34, 321 (1973).

2. G.A. Slack and T.F. McNelly, J. Cryst. Growth 34, 263 (1976).

3. G.A. Slack and T.F. McNelly, J. Cryst. Growth 42, 560 (1977).

4. L.M. Sheppard, Ceram. Bull. 69, 1801 (1990).

5. B. Hejda and K. Hauptmanová, Phys. Status Solidi 36, K95 (1969).

6. D. Jones and A.H. Lettington, Solid State Commun. 11, 701 (1972).

7. A. Kobayashi, O.F. Sankey, S.M. Volz, and J.D. Dow, Phys. Rev. B 28, 935 (1983).

8. M-Z. Huang and W-Y. Ching, J. Phys. Chem. Solids 46, 977 (1985).

9. W-Y. Ching and B.N. Harmon, Phys. Rev. B 34, 5305 (1986).

10. R.V. Kasowski and F.S. Ohuchi, Phys. Rev. B 35, 9311 (1987).

11. Y-N. Xu and W-Y. Ching, Phys. Rev. B 48, 4335 (1993).

12. E. Ruin, S. Alvarez, and P. Alimony, Phys. Rev. B 49, 7115 (1994).

13. A.K. Saloon, A. Kashyap, T. Nautiyal, and S. Auluck, Solid State Commun. 94, 1009 (1995).

14. F.S. Ohuchi, J. Phys. 49(10), C5-783 (1988); J. Appl. Phys. 62, 2286 (1987).

15. P.F. Carcia, R.H. French, K. Sharp, J.S. Meth, and B.W. Smith, Proc. SPIE-Int. Soc. Opt. Eng. 2884, 255 (1996).

16. P.F. Carcia, R.H. French, M.H. Reilly, M.F. Lemon, and D.J. Jones, Appl. Phys. Lett. 70, 2371 (1997).

17. L.E. McNeil, M. Grimsditch, and R.H. French, J. Am. Ceram. Soc. 76, 1132 (1993).

18. M. Morita, K. Tsubouchi, and N. Mikoshiba, Jpn. J. Appl. Phys. 21, 1102 (1982).

19. G. Radhakrishnan, J. Appl. Phys. 78, 6000 (1995).

20. H. Yamashita, K. Fukui, S. Misawa, and S. Yoshida, J. Appl. Phys. 50, 896 (1979).

21. T.L. Chu and R.W. Elm, Jr., J. Electrochem. Soc. 122, 995 (1975).

22. C.R. Aita, C.J.G. Kubiak, and F.Y.H. Shih, J. Appl. Phys. 66, 4360 (1989).

23. R. Zarwasch, E. Rille, and H.K. Pulker, J. Appl. Phys 71, 5275 (1992).

24. J.C. Kaplan and R.A. Gerhardt, J. Mater. Res. 9, 2209 (1994)

25. P.B. Perry and R.F. Rutz, Appl. Phys. Lett. 33, 319 (1978).

26. J. Bauer, L. Biste, and D. Bolze, Phys. Status Solidi A 39, 173 (1977).

27. N.V. Barovskii, U.D. Kudakov, E.B. Sokolov, and V.G. Sredin, Izv. Akad. Nauk SSSR, Neorg. Mater. 19, 1589-1591 (1983) [translation by Plenum Publishing (1984)].

28. Q. Guo and A. Yoshida, Jpn. J. Appl. Phys. 33, 2453 (1994). 
29. G.A. Slack, Advanced Materials for Optical Windows (General Electric Technical Information Series No. 79CRD071, under ONR Contract N00014-77-C-0649, Schenectady, NY, June 1979).

30. V.N. Meleshkin, V.V. Mikhailin, V.E. Oranovskii, P.A. Orekhanov, I. Pasternák, S. Pacesova, A.S. Salamatov, M.V. Fok, and A.S. Yarov, in Synchrotron Radiation, edited by N.G. Basov [Proceedings (Trudy) of the P.N. Lebendev Physics Inst., Moscow, c. 1975], Vol. 80, pp. 169-174.

31. V.V. Michailin, V.E. Oranovskii, S. Pacesová, J. Pastrnák, and A.S. Salamatov, Phys. Status Solidi B 55, K51 (1973).

32. M. Gautier, J.P. Duraud, and C. LeGressus, J. Appl. Phys. 61, 574 (1987).

33. C.G. Olson, J.H. Sexton, D.W. Lynch, A.J. Bevolo, H.R. Shanks, B.N. Harmon, W.Y. Ching, and D.M. Wieliczka, Solid State Commun. 56, 35 (1985).

34. A.D. Dorneich, R.H. French, H. Müllejans, S. Loughin, and M. Rühle, J. Microsc. 191(3), 286-296 (1988); R.H. French, H. Müllejans, D.J. Jones, J. Am. Ceram. Soc. 81, 2549 (1998).

35. S. Loughin and R.H. French, in Properties of Group III Nitrides, edited by J.H. Edgar (Electronic Materials Information Service, INSPEC, Institution of Electrical Engineers, London, 1994), pp. 175-189.

36. S. Loughin, R.H. French, in Handbook of Optical Constants of Solids, edited by E. Palik (Academic Press, New York, 1998), Vol. III, pp. 373-401.

37. S. Loughin, R.H. French, W.Y. Ching, Y.N. Xu, and G.A. Slack, Appl. Phys. Lett. 63, 1182 (1993).

38. Shin-Etsu Chemical Co., Limited, Advanced Materials Division, 6-1, 2 Chome Otemachi Chiyoda-ku, Tokyo.

39. B. Johs, R.H. French, F.D. Kalk, W.A. McGahan, and J.A. Woollam, Proc. SPIE-Int. Soc. Opt. Eng. 2253, 1098 (1994).

40. M.L. Bortz and R.H. French, Appl. Phys. Lett. 55, 1955 (1989).

41. R.H. French, Phys. Scr. 41(4), 404 (1990).

42. D.Y. Smith, in Handbook of Optical Constants of Solids, edited by E.D. Palik (Academic Press, New York, 1985), pp. 35-68.

43. S. Loughin, R.H. French, L.K. DeNoyer, W-Y. Ching, and Y-N. Xu, J. Phys. D 29, 1740 (1996).

44. R. de L. Kronig, J. Opt. Soc. Am. 12, 547 (1926); C.J. Gorter and R. de L. Kronig, Phys. III 9, 1009 (1936).

45. H.A. Kramers, Atti. Congr. Int. Fis. Como. 2, 545 (1927).

46. M.L. Bortz and R.H. French, Appl. Spectrosc. 43, 1498 (1989).

47. W. Sellmeier II. Ann. Phys. Chem. 147, 525 (1872) (in German).

48. S. Jacoby, J. Kowalik, and J. Pizzo, Iterative Methods for NonLinear Optimization Problems (Prentice-Hall, Englewood Cliffs, NJ, 1972).

49. F. Wooten, Optical Properties of Solids (Academic Press, San Diego, CA, 1972).

50. Duplex II Refractometer, Gem Instruments Corp., P.O. Box 2147, 1630 Stewart St., Santa Monica, CA 90406.

51. T. Tomiki, Y. Ganaha, T. Shikenbaru, T. Futemma, M. Yuri, Y. Aiura, S. Sato, H. Fukutani, H. Kato, T. Miyahara, A. Yonesu, and J. Tamashiro, J. Phys. Soc. Jpn. 62, 573 (1993).

52. T. Tomiki, Y. Ganaha, T. Shikenbaru, T. Futemma, M. Yuri, Y. Aiura, S. Sato, H. Fukutani, H. Kato, T. Miyahara, A. Yonesu, and J. Tamashiro, J. Phys. Soc. Jpn. 62, 1372 (1993).

\section{APPENDIX: KRAMERS-KRONIG ANALYSIS}

The mathematical basis for the analysis of reflectance data to obtain any conjugate pair of complex optical properties was established by Kronig ${ }^{44}$ and independently claimed by Kramers. ${ }^{45}$ Their theoretical treatment provides an explicit prescription for determining one optical property from its conjugate partner but requires knowledge of that partner spanning an infinite energy range. In practice, the scope of the experimental data is limited and must be extended by extrapolation methods. But the nature of the transform method implies that errors in the extrapolation of a property outside the measured range lead to errors in the determined conjugate property within the measured range. Also, sample condition and geometrical factors in the optical system introduce systematic errors in the amplitude of the measured data. Because slope discontinuities in the measured property lead to features in the calculated optical property, the particulars of the method for extrapolation and amplitude correction can have a significant impact on the validity of the result. For these reasons we set forth in this Appendix a concise description of the analysis methods employed in the foregoing presentation.

\section{A. KK Analysis of reflectance data}

Given knowledge of the reflected amplitude for all photon energies, $\rho(E)=\sqrt{R(E)}$, Kramers-Kronig (KK) analysis prescribes a transform method to recover the phase $\theta(E)$ of the reflected wave, specifically

$$
\theta(E)=-\frac{2 E}{\pi} P \int_{0}^{\infty} \frac{\ln \left\{\rho\left(E^{\prime}\right)\right\}}{E^{\prime 2}-E^{2}} \mathrm{~d} E^{\prime} .
$$

From the amplitude and phase, any conjugate pair of optical properties can be computed. The complex index of refraction, for example, is obtained by solving the relation

$$
\frac{n-1+i k}{n+1+i k}=\rho(E) \mathrm{e}^{i \theta(E)}
$$

\section{B. KK analysis method}

In the present work, we employ a spectral method ${ }^{46}$ based on fast-Fourier transformation (FFT) of the data to realize a numerical solution for Eq. A1. Before applying this method, it is necessary to properly scale the amplitude of the measured data and provide extrapolations for the low- and high-energy ranges not covered by the measurement, commonly called "wings."

\section{Amplitude correction and low-energy extrapolation}

Typically, the scaling of reflectance data is accomplished by selecting a literature value for the index of refraction in the visible range and then scaling the entire data set to agree with the reflectance computed from this value. However, this relies on the validity of very few points or even a single point in the measured data.

We employ multiple data sources, specifically spectroscopic ellipsometry and UV/VIS spectroscopy results, 
to determine the optical properties in the UV/VIS range for thin film AlN. The refined model of the optical constants for AlN film \#8346 provides reliable values for $R(E)$ over an extended energy range, with which we can both scale and extend the VUV/UV data below the experimental energy range of the VUV instrument. The scaling and low-energy extrapolation procedure is as follows. The VUV data are scaled by a factor estimated to compensate for any geometrical imaging errors; in this work it was 1.55 to compensate for the small size of the AlN single crystal. Then a wing is prepared at the lowenergy side of the VUV range where absorption is minimal $(k \sim 0)$, using a two-pole Sellmeier equation ${ }^{47}$ :

$n(\lambda)=\sqrt{A+\sum_{i=1}^{2} \frac{B_{i} \lambda^{2}}{\lambda^{2}-C_{i}^{2}+\left(E_{i} \lambda / \lambda-C_{i}\right)^{2}}}$,

where $A$ is a constant, the $B_{i}$ are the amplitudes, the $C_{i}$ are the wavelength positions, and the $E_{i}$ are the widths of the two poles. Since absorption is assumed to be negligible, reflectance data are fit to $(n(\lambda)-1)^{2}(n(\lambda)+1)^{-2}$, to determine these Sellmeier parameters using the Simplex method. ${ }^{48}$ The wing is simultaneously fit to the first $1 \mathrm{eV}$ range of the scaled VUV data, in this case from $E_{1}<$ $E<\left(E_{1}+1\right)$ or about $2-3 \mathrm{eV}$, and also to the reflectance calculated from ellipsometry data over any chosen range or for specific points, in this case from 1.5 to $4 \mathrm{eV}$. This procedure is iterated using different scale factors for the VUV data to optimize the fit of the Sellmeier wing to the reflectance determined from VUV and ellipsometry data, by minimizing the error metric, $\chi^{2}$. This provides a reproducible way to simultaneously find the appropriate scale factor for the experimental reflectance data and extend the VUV data down to zero energy using the Sellmeier wing in a manner that matches both the amplitude and slope of the experimental data at the $E_{1}$ limit. Augmenting the VUV data in this way improves the accuracy of the VUV data scaling and also improves the fidelity of the low-energy wing extension.

\section{High-energy extrapolation}

VUV spectroscopy is well established as a method for determining the optical properties and the electronic structure of wide band gap insulators. The data obtained in this method generally span the full range of valence to conduction band transitions. In the high-energy limit the valence electrons behave as a nearly-free electron gas. A Lorenz-oscillator model predicts that the reflectance of such a gas obeys an inverse-fourth-power law, ${ }^{49}$ as shown in Table A-I. Consistent with this model for the high-energy optical response, the data should be extended by an expression of the form $A E^{-4}$ with the prefactor $A$ obtained by a linear regression fit to the high-energy segment of the experimental data, typically over the last $2 \mathrm{eV}$. The high-energy wing extends to the high energy cutoff, $E_{\mathrm{hc}}$. Generally $E_{\mathrm{hc}}=1000 \mathrm{eV}$ is a reasonable choice since the oscillator strength in the wing above this value is negligible. A detailed comparison with optical constants determined by EELS, a method that probes transitions from both valence and core levels, supports the use of the $E^{-4}$ model as essential to accurate analysis, giving the closest agreement between VUV and EELS results. ${ }^{34}$

The methods employed here are versatile and do not require the use of literature values for the index of refraction for low-energy extrapolation. The results are more accurate and minimize the possibility of artifacts due to data preparation errors. We next discuss how they differ from our own earlier approach, and that employed by other groups of investigators.

\section{Discussion of KK methods}

In previous investigations, the low- and high-energy wing preparation methods employed by the authors were simpler and are summarized in Table A-II. Typically, the reflectance was scaled to match the index of refraction at a single wavelength in the visible, usually $633 \mathrm{~nm}$, relying on data from the literature or a gemologists refrac-

TABLE A-I. Power laws for the optical properties in the limit of high energies, where $\omega_{\mathrm{p}}=\sqrt{4 \pi n e^{2} m_{\mathrm{o}}^{-1}}$ is the plasma frequency, $n$ the electron density, and $\Gamma$ the damping constant.

\begin{tabular}{lc}
\hline \hline Optical property & Power law \\
\hline $\begin{array}{c}\text { Complex dielectric constant } \\
\epsilon_{1}+i \epsilon_{2}\end{array}$ & $\epsilon_{1}=1-\frac{\omega_{\mathrm{p}}^{2}}{\omega^{2}}$ \\
Reflectance $R(\omega)$ & $\epsilon_{2}=\frac{\omega_{\mathrm{p}}^{2} \Gamma}{\omega^{3}}$ \\
& $R(\omega)=\frac{\omega_{\mathrm{p}}^{4}}{16 \omega^{4}}$ \\
\hline
\end{tabular}

TABLE A-II. Reflectivity preparation for Kramers-Kronig dispersion analysis.

\begin{tabular}{llrl}
\hline \hline & \multicolumn{1}{c}{ Low-energy wing } \\
$E<E_{1}$ & Experimental data range & $E_{1}$ to $E_{\mathrm{h}}$ & $\begin{array}{c}\text { High-energy wing } \\
E>E_{\mathrm{h}}\end{array}$ \\
\hline Improved method & Two pole Sellmeier fit to ellipsometric optical constants & Multiplicative scaling & $A E^{-4}$ \\
Prior method ${ }^{37}$ & Polynomial & Multiplicative scaling & $A E^{-p}, p$ variable \\
Tomiki's method $^{51,52}$ & Three pole Sellmeier & Multiplicative scaling & $A E^{-p}, p$ variable \\
\hline \hline
\end{tabular}


tometer. ${ }^{50}$ The reflectance was calculated by assuming negligible absorption $(k \approx 0)$ so $R(E)=(n-1)^{2}$ $(n+1)^{-2}$.

A simple polynomial low-energy wing was anchored to this reflectance point, and the scale factor applied to the experimental data was determined so as to join the wing and the data with a continuous slope. The highenergy wing, modeled as $E^{-p}$, would be attached next. A range of ten $p$ values was used to calculate optical properties, and the results were reviewed to identify the most reasonable result; for example, absorption might be negative for some values of $p$. Typically a high-energy wing exponent in the range $6<p<7$ was selected. The fact that this exponent exceeded a value of 4 tended to overemphasize features in the absorptive optical properties near the band gap, such as $\operatorname{Re}\left[J_{\mathrm{cv}}\right]$, the optical conductivity $\left(\sigma_{1}\right)$, and the dielectric constant $\left(\epsilon_{2}\right)$. At the same time the amplitude of features near the highenergy cutoff was diminished. The integrated area for the spectral line shapes was conserved, in that these amplitude differences were not easily discerned by calculating the oscillator-strength sum rule over the entire range.

Other investigators ${ }^{51,52}$ have also used a $E^{-p}$ power law where $p$ is selected so that the spectral behavior of the absorption constant derived from $R(E)$ by KK analysis conforms reasonably well to the Urbach rule. Instead of varying the exponent $p$, the method presented here assumes $p$ is always 4 , consistent with free electron behavior, and the principal adjustment to the data is scaling it to fit the VUV data to the ellipsometry data on the low-energy side. 